\title{
On the Euler Characteristic of Finite Unions of Convex Sets
}

\author{
Beifang Chen \\ Department of Mathematics, Massachusetts Institute of Technology, \\ Cambridge, MA 02139, USA \\ bchen@math.mit.edu
}

\begin{abstract}
The Euler characteristic plays an important role in many subjects of discrete and continuous mathematics. For noncompact spaces, its homological definition, being a homotopy invariant, seems not as important as its role for compact spaces. However, its combinatorial definition, as a finitely additive measure, proves to be more applicable in the study of singular spaces such as semialgebraic sets, finitely subanalytic sets, etc. We introduce an interesting integral by means of which the combinatorial Euler characteristic can be defined without the necessity of decomposition and extension as in the traditional treatment for polyhedra and finite unions of compact convex sets. Since finite unions of closed convex sets cannot be obtained by cutting convex sets as in the polyhedral case, a separate treatment of the Euler characteristic for functions generated by indicator functions of closed convex sets and relatively open convex sets is necessary, and this forms the content of this paper.
\end{abstract}

\section{An Euler Integral}

Given a function $\chi$ on the class of all compact convex sets of a Euclidean space $\mathbb{R}^{n}$ with constant value 1 , Hadwiger [8] uniquely extended $\chi$ to the class of finite unions of compact convex sets by finite additivity. Groemer [3], using a similar idea, considered the vector space $V(\mathscr{K})$ of functions generated by indicator functions of compact convex sets of $\mathbb{R}^{n}$, and uniquely extended $\chi$ to a linear functional on $V(\mathscr{K})$. The indicator function, or simply indicator, of a subset $A$ of $\mathbb{R}^{n}$ is the function $1_{A}$ such that $1_{A}(x)=1$ for $x \in A$ and $1_{A}(x)=0$ for $x \notin A$. Groemer's vector space obviously contains Hadwiger's convex ring of finite unions of compact convex sets. Moreover, it has been shown by Groemer that the Minkowski sum, i.e., vector addition, induces uniquely a commutative multiplication on $V(\mathscr{K})$ which plays an important role in combinatorial geometry, 
where $\mathscr{K}$ is the class of all compact convex sets in Euclidean space. Both Hadwiger and Groemer used induction on the dimension of spaces by applying projection to derive the Euler characteristic for their objects. More recently, Chen [2] derived the duality between the Gram-Sommerville and Gauss-Bonnet formulas for polyhedra by using the Euler characteristic; and Schanuel [18] gave a classification of polyhedra up to polyhedral isomorphism by two invariants: an Euler characteristic in $\mathbb{Z} \times \mathbb{Z}$, and a "dimension" in order ideals of a certain partially ordered set. For unbounded polyhedra, the first component Euler characteristic is also shown to be well defined in [2] by cutting unbounded relatively open polytopes. In Schanuel's classification of polyhedra, his Euler characteristic is naturally obtained for arbitrary closed convex sets and relatively open convex sets. The first component of $\mathbb{Z} \times \mathbb{Z}$ is of most interest to us because it is a topological invariant, while the second component is an approximation extension by compact convex sets, see [18]. Morelli [13] independently also obtained the second component Euler characteristic for unbounded polyhedra. The second Euler characteristic can be considered as a special case of the Euler characteristic of the Klee-Rota type [10], [15] for lattices, or, more generally, for partially ordered sets, see also [14]. For other indirectly related work, see the excellent references of [12] and [1] and [7]. We share the ideas, more or less, of Groemer [3], [5], Hadwiger [8], Rota [15], [16], and Schanuel [17], [18], from a different point of view by introducing an integral as follows.

Definition 1.1. Let $r$ and $s$ be two fixed real numbers such that $r+s=1$. For a real-valued function $f(x)$ on $\mathbb{R}$, set

$$
\int f(x) d x=\sum_{x \in \mathbb{B}}\left(f(x)-r f\left(x^{-}\right)-s f\left(x^{+}\right)\right),
$$

where $\mathbb{R}=\mathbb{R} \cup\{\infty\}, f(\infty)=0, f\left(x^{-}\right)$and $f\left(x^{+}\right)$denote the left and right limits of $f$ near $x \in \mathbb{R}$, respectively, $f\left(\infty^{-}\right)=\lim _{x \rightarrow+\infty} f(x)$, and $f\left(\infty^{+}\right)=\lim _{x \rightarrow-\infty} f(x)$. $\int f d x$ is called the Euler integral of $f$; and $f$ is called the Euler integrable if $f(x)$ is continuous except at finitely many points, and has both left and right limits at those discontinuous points.

Definition 1.2. Let $v=\left\{v_{1}, \ldots, v_{n}\right\}$ be an ordered basis of a Euclidean $n$-space $\mathbb{R}^{n}$. The Euler integral of a function $f(x)$ on $\mathbb{R}^{n}$ is defined to be the formal iterated integral

$$
\begin{aligned}
\chi(f, u) & =\int \cdots \int f d v_{1} \cdots d v_{n} \\
& =\int f\left(T_{v}\right) d x_{1} \cdots d x_{n},
\end{aligned}
$$

where $T_{v}$ is the linear transformation of $\mathbb{R}^{n}$ which maps the standard basis 
$e=\left\{e_{1}, \ldots, e_{n}\right\}$ to $v$, all the components of $e_{i}$ are zero except 1 at the $i$ th position, $1 \leq i \leq n . f(x)$ is called the Euler integrable with respect to the basis $v$ if each of its iterated Euler integrals is Euler integrable; and $f(x)$ is simply called Euler integrable if it is so for all bases.

The idea of the above definition is implicitly used by Hadwiger [8] for $r=1$ and by Groemer [3] for $r=s=\frac{1}{2}$, but the use of the iterated integral process is due to Schanuel for $r=s=\frac{1}{2}$, via lectures at SUNY-Buffalo, and is actually a special case of his definition of curvature measures. The iterated integral process is also due to Rota [16] for the case $r=1$.

Theorem 1.3. For any fixed real numbers $r$ and $s$ such that $r+s=1$, the set $\mathscr{E}_{r, s}\left(\mathbb{R}^{n}\right)$ of all Euler integrable functions on $\mathbb{R}^{n}$ with the chosen numbers $r$ and $s$ is a vector space over $\mathbb{R}$.

Proof. $\mathscr{E}_{r, s}\left(\mathbb{R}^{n}\right)$ is obviously nonempty. For any Euler integrable functions $f$ and $g$, i.e., all their iterated Euler integrals are continuous about each coordinate except at finitely many points and they have both left and right limits at those discontinuous points, it is obvious that the iterated integrals of $f+g$ and $a f$, for a real number $a$, are continuous about each coordinate except at finitely many points and they have left and right limits at those discontinuous points. Then $f+g$ and af are also Euler integrable. So $\mathscr{E}_{r, s}\left(\mathbb{R}^{n}\right)$ is a vector space over $\mathbb{R}$.

It may be tedious to state the so simple result because it is a straightforward consequence of our definition. However, it is this vector property that shows the advantage of our definition in avoiding decomposition and extension as in the traditional treatment of the combinatorial Euler characteristic. It should be noticed that in general $\mathscr{E}_{r, s}\left(\mathbb{R}^{n}\right)$ is not closed under pointwise multiplication of functions.

\section{The Euler Characteristic}

In this section we show that the indicator functions of (unbounded) polyhedra, closed polyconvex sets, and relatively open polyconvex sets of $\mathbb{R}^{n}$ are all Euler integrable. There are many other sets of $\mathbb{R}^{n}$ whose indicator functions are Euler integrable, for example, semialgebraic sets and finitely subanalytic sets, and we treat them elsewhere by different techniques. For this reason, we give a definition of the Euler characteristic for sets of $\mathbb{R}^{n}$ whose indicator functions are Euler integrable.

Theorem 2.1. For a subset $E$ of $\mathbb{R}^{n}$, if its indicator function is Euler integrable, then the Euler integral of its indicator function with respect to any basis is always an integer, and it is independent of $r$ and $s$ such that $r+s=1$. 
Proof. We apply induction on the dimension $n$. For $n=1$, an Euler set $E$ of $\mathbb{R}$ has to be a disjoint union of finitely many open intervals and singleton sets. For an open interval $(a, b)$ and a singleton $\{c\}$, we have

$$
\begin{aligned}
& \int 1_{(a, b)} d x=(0-s)+(0-r)=-1, \\
& \int 1_{\{c\}} d x=1 .
\end{aligned}
$$

So the Euler integral of $1_{E}$ is a sum of some 1 and -1 which is independent of $r$ and $s$. Suppose it is true for Euler sets in a Euclidean space of dimension $n-1$. For any basis $v=\left\{v_{1}, \ldots, v_{n}\right\}$ of $\mathbb{R}^{n}$, consider $1_{E}$ as a function $f_{E}$ on $\mathbb{R}^{n}$ under the coordinates of $v$, i.e.,

$$
f_{E}\left(x_{1}, \ldots, x_{n}\right)=1_{E}\left(x_{1} v_{1}+\cdots+x_{n} v_{n}\right)
$$

For each $x_{n}, f_{E}\left(\cdot, x_{n}\right)$ defines a function on $\mathbb{R}^{n-1}$. Obviously, $f_{E}\left(\cdot, x_{n}\right)$ is Euler integrable over $\mathbb{R}^{n-1}$. It is Euler integrable especially with respect to the basis $\left\{v_{1}, \ldots, v_{n-1}\right\}$. Then

$$
\begin{aligned}
f(x) & =\int f_{E}(\cdot, x) d x_{1} \cdots d x_{n-1} \\
& =\int 1_{E} d v_{1} \cdots d v_{n-1}
\end{aligned}
$$

defines a function on $\mathbb{R}$ which is also Euler integrable. By induction hypothesis, $f(x)$ is integer valued, so $f(x)$ can be written as a finite sum

$$
f(x)=\sum \alpha_{i} 1_{\left(a_{i}, b_{i}\right)}+\sum \beta_{j} 1_{\left[c_{j}\right]}
$$

where $\alpha_{i}$ and $\beta_{j}$ are integers and independent of $r$ and $s$. Thus

$$
\int 1_{E} d v_{1} \cdots v_{n}=\int f(x) d x
$$

is an integer, and it is independent of $r$ and $s$ such that $r+s=1$.

Definition 2.2. A subset $E$ of $\mathbb{R}^{n}$ is called an Euler set if its indicator function $1_{E}$ is Euler integrable. If the Euler integrals of $1_{E}$ with respect to all bases of $\mathbb{R}^{n}$ are the same, then the value is called the Euler characteristic of $E$, and is denoted by $\chi(E)$. 
We hope, for any Euler set $E$, its Euler integral with respect to any basis is the same. Although we cannot prove this for all such sets, we can show it is true for some sets of this kind. As in the structure theorem of closed convex sets stated in Section 3, a closed convex set $F$ has one of the following three forms:

(1) $F$ is compact,

(2) $F=K \oplus V^{k}$, where $K$ is a compact convex set and $V^{k}$ is a nonzero $k$-dimensional subspace of $\mathbb{R}^{n}$, and

(3) $F=M \oplus W \oplus L$, where $W$ is a vector subspace, $M$ is a topological manifold (with or without boundary) which is contained in the boundary $\partial \pi(F)$ of $\pi(F)$, where $\pi$ is the canonical projection of $\mathbb{R}^{n}$ onto $\mathbb{R}^{n} / W$, and $L$ is a closed half-line.

By this classification we obtain the following theorem.

Theorem 2.3. Closed convex sets and relatively open convex sets of $\mathbb{R}^{n}$ are Euler sets. So the vector space spanned by indicator functions of those sets is a subspace of $\mathscr{E}_{r, s}\left(\mathbb{R}^{n}\right)$. Moreover, for a nonempty relatively open convex set $U$ and a nonempty closed convex set $F$ :

1. $\chi(U)=(-1)^{\operatorname{dim}(U)}$.

2. $\chi(F)=1$ whenever $F$ is compact.

3. $\chi(F)=(-1)^{k}$ whenever $F=K \oplus V^{k}$.

4. $\chi(F)=0$ whenever $F=M \oplus E \oplus L$.

Proof. As the proof of Theorem 2.1 shows, the Euler integrals of the indicator functions of an open interval and a singleton set of $\mathbb{R}$ are -1 and 1 , respectively. Therefore the Euler integral of an indicator function of any closed interval $[a, b]$ is 1 because $1_{[a, b]}=1_{\{a\}}+1_{(a, b)}+1_{\{b\}}$. It can be easily checked that the Euler integral of any unbounded open interval is also -1 . The proof proceeds by induction on the dimension of spaces.

For dimension 1, it is true as stated above. Suppose it is true for dimension $n-1$. Now, for dimension $n$, let $v=\left\{v_{1}, \ldots, v_{n}\right\}$ be a basis of $\mathbb{R}^{n}$, let $L_{i}$ denote the subspace spanned by $v_{i}, 1 \leq i \leq n$, and let $\pi$ be the projection of $\mathbb{R}^{n}$ onto $X=L_{2} \oplus \cdots \oplus L_{n}$ whose kernel is $L_{1}$. We first consider a nonempty relatively open convex set $U$. Note that $\pi(U)$ is also a relative open convex set and, for every point $p \in \pi(U)$, the intersection $\left(p+L_{1}\right) \cap U$ is simultaneously an open interval or a singleton. Then we have

$$
\int 1_{U} d v_{1} \cdots d v_{n}=\delta \int 1_{\pi(U)} d v_{2} \cdots d v_{n},
$$

where $\delta=-1$ if $\operatorname{dim} \pi(U)=\operatorname{dim}(U)-1$ and $\delta=1$ if $\operatorname{dim} \pi(U)=\operatorname{dim}(U)$. By induction we have

$$
\chi(U)=\int 1_{U} d v_{1} \cdots d v_{n}=(-1)^{\mathrm{dim}(U)}
$$


Let $F$ be a nonempty closed convex set. If $F$ is compact, then $\left(p+L_{1}\right) \cap F$ is a closed segment or a singleton for all $p \in \pi(F)$, and $\pi(F)$ is a compact convex set in $X$. So, by the induction hypothesis,

$$
\chi(F)=\int 1_{F} d v_{1} \cdots d v_{n}=1
$$

If $F$ is not compact, i.e., $F$ is unbounded, we have two cases: $F=K \oplus V^{k}$ and $F=M \oplus W \oplus L$. For the case $F=K \oplus V^{k}$, if $v_{1} \in V^{k}$, then $\pi(F)=\pi(K) \oplus V^{k-1}$, where $V^{k-1}$ is a $(k-1)$-dimensional subspace of $X$. Thus by induction

$$
\begin{aligned}
\int 1_{F} d v_{1} \cdots d v_{n} & =-\int 1_{\pi(F)} d v_{2} \cdots d v_{n} \\
& =-(-1)^{k-1} \\
& =(-1)^{k} .
\end{aligned}
$$

If $v_{1} \notin V^{k}$, we have $\pi(F)=K_{1} \oplus V_{1}^{k}$, where $K_{1}$ is a compact convex set of $X, V_{1}^{k}$ is a nonzero $k$-dimensional subspace of $X$, and $\pi^{-1}(p) \cap F$ is a bounded closed segment for all $p \in \pi(F)$. Then by induction

$$
\int 1_{F} d v_{1} \cdots d v_{n}=\int 1_{\pi(F)} d v_{2} \cdots d v_{n}=(-1)^{k}
$$

For the case $F=M \oplus W \oplus L$, if $L_{1} \subset W$, then $\pi(F)=M^{\prime} \oplus W^{\prime} \oplus L^{\prime}$, where $M^{\prime}$ is a topological manifold with or without boundary, $W^{\prime}$ is a vector subspace of $W$ such that $\operatorname{dim}\left(W^{\prime}\right)=\operatorname{dim}(W)-1$, and $L^{\prime}$ is a closed half-line, thus by induction we have

$$
\int 1_{F} d v_{1} \cdots d v_{n}=-\int 1_{\pi(F)} d v_{2} \cdots d v_{n}=0
$$

If there exists a closed half-line $L_{1}^{\prime} \subset L_{1}$ such that $F=M_{1} \oplus W \oplus L_{1}^{\prime}$, then, for each $p \in \pi(F),\left(p+L_{1}\right) \cap F$ is a closed half-line. Thus $\chi(F)=\int 1_{F} d v_{1} \cdots d v_{n}=0$. Otherwise, we claim that $\pi(F)$ is a closed unbounded convex subset of $X$, the fiber $\pi^{-1}(p) \cap F=\left(p+L_{1}\right) \cap F$ is a bounded closed segment for each $p \in \pi(F)$, and $\pi(F)=M^{\prime} \oplus W^{\prime} \oplus L^{\prime}$ has the form (3).

The convexity of $\pi(F)$ is obvious. For boundedness of $\pi^{-1}(p) \cap F$, suppose there is one point $q \in \pi(F)$ such that the closed set $\pi^{-1}(q) \cap F$ is unbounded, that is, it is a closed half-line, say $\left\{q+t v_{1} \mid t \geq t_{0}\right\}$ for some $t_{0} \in \mathbb{R}$. Then, for any $p \in \pi(F)$, $p+s_{0} v_{1}$ belongs to $\pi^{-1}(p) \cap F$ for some $s_{0} \in \mathbb{R}$, here $s_{0}$ is chosen to be smallest. Thus the segment $\left\{s\left(p+s_{0} v_{1}\right)+(1-s)\left(q+t v_{1}\right) \mid 0 \leq s \leq 1\right\}$ from $p+s_{0} v_{1}$ to $q+t v_{1}$ is contained in $F$ for all $t \geq t_{0}$. Let $t$ tend to $\infty$, then the segments tend to the closed half-line $\left\{p+t v_{1} \mid t \geq s_{0}\right\}$ which should be $\pi^{-1}(p) \cap F$. This becomes the former case. Thus $\pi^{-1}(p) \cap F$ is a bounded segment for all $p \in \pi(F)$. 
For closedness of $\pi(F)$, let $\left\{p_{k}\right\}$ be a sequence in $\pi(F)$ such that $p_{k}$ converges to $p$. Let $\left\{q_{k} \in \pi^{-1}\left(p_{k}\right)\right\}$ be a corresponding sequence in $F$. It can be checked that $\left\{q_{k}\right\}$ is bounded. Then $\left\{q_{k}\right\}$ has a subsequence $\left\{q_{k}\right\}$ which converges to $q$. Thus $p_{k_{i}}=\pi\left(q_{k}\right)$ converges to $\pi(q)=p$ as $k_{j}$ tends to $\infty$. So $\pi(F)$ is closed. Note that $\pi(F)$ is also unbounded, otherwise $\pi(F)$ will be compact, and this implies that $F$ is compact, which is a contradiction. Since $\pi(F)$ contains the half-line $\pi(L)$, but not the whole line, then $\pi(F)=M^{\prime} \oplus W^{\prime} \oplus L^{\prime}$ has the form (3). Therefore by induction

$$
\int 1_{F} d v_{1} \cdots d v_{n}=\int 1_{\pi(F)} d v_{2} \cdots d v_{n}=0 .
$$

Let the vector space spanned by the indicator functions of closed convex sets and relatively open convex sets of $\mathbb{B}^{n}$ be denoted by

$$
V(\mathscr{F} \cup \mathcal{O})=\left\{\sum_{i} a_{i} 1_{F_{1}}+\sum_{j} b_{j} 1_{U_{J}}\right\},
$$

where $a_{i}, b_{j} \in \mathbb{R}, F_{i}$ is a closed convex set, $U_{j}$ is a relatively open convex set, and $\mathscr{F}$ and $\mathcal{O}$ denote the class of closed convex sets and the class of relatively open convex sets, respectively. If we identify a set of $\mathbb{R}^{n}$ with its indicator function, then $V(\mathscr{F} \cup \mathcal{O})$ certainly contains the sets which are finite unions of closed convex sets and the sets which are finite unions of relatively open convex sets because, for any subsets $E_{i}(i=1, \ldots, m)$,

$$
1_{E_{1} \cup \cdots \cup E_{m}}=\sum_{k=1}^{m}(-1)^{k-1} \sum_{i_{1}<\cdots<i_{k}} 1_{E_{i}, \cdots \cap E_{i k}} .
$$

For example, any polyhedron is in it, and we immediately have Euler's formula: for a nonempty convex polytope $P$, let $\alpha_{i}$ be the number of $i$-faces of $P$, then $\sum_{i}(-1)^{i} \alpha_{i}=1$. Furthermore, $V(\mathscr{F} \cup \mathcal{O})$ contains any convex surface $\partial F$ of a closed convex set $F$ and any polyhedron on $\partial F$ since $\partial F=F-\operatorname{Int}_{r}(F)$, where $\operatorname{Int}_{r}(F)$ is the relative interior of $F$. A convex surface polyhedron on $\partial F$ is a finite union of relatively open surface polytopes on $\partial F$. A relatively open surface polytope on $\partial F$ is a nonempty intersection of $\partial F$ and a relatively open convex polyhedral cone with apex $p \in \operatorname{Int}_{r}(F)$ such that if two open rays $p+L_{1}$ and $p+L_{2}$ in the cone emanating from the point $p$ meet $\partial F$, then any open ray in $p+L_{1} \oplus L_{2}$ emanating from $p$ meets $\partial F$. Indeed, let $C$ be a closed convex polyhedral cone in the flat that $F$ spans with apex $p \in \operatorname{Int}_{r}(F)$. Note that the boundary $\partial C$ is in $V(\mathscr{F} \cup \mathcal{O})$. If $\partial C$ is written as $\partial C=C_{1} \cup \cdots \cup C_{k}$, where $C_{1}, \ldots, C_{k}$ are facets of $C$, then $(\partial C) \cap F=\left(C_{1} \cap F\right) \cup \cdots \cup\left(C_{k} \cap F\right)$ is in $V(\mathscr{F} \cup \mathcal{O})$. Thus

$$
F \cap C-F \cap \partial C-\operatorname{Int}_{r}(F \cap C)
$$

is in $V(\mathscr{F} \cup \mathcal{O})$, and it is a relatively open surface polytope on $\partial F$. So we have Euler's formula on convex surfaces. 
Corollary 2.4. Let $P$ be a nonempty closed bounded polytope on a convex surface, and let $\alpha_{i}$ be the number of $i$-faces of $P$, then $\sum_{i}(-1)^{i} \alpha_{i}=1$.

The above corollary is a generalization of the spherical Euler relation of Hadwiger and Mani [9], it is also a generalization of Groemer's Euler relation for polytopes on convex surfaces of compact convex sets [4]. Our convex surface can be the boundary of any closed convex set. It should be noted that our unified treatment of Euler characteristic on $V(\mathscr{F} \cup \mathcal{O})$ includes all the Euler characteristics studied in [1]-[6], [8]-[10], and [12]-[18] and more geometric objects. It should be noticed that $V(\mathscr{F} \cup \mathcal{O})$ is not a ring under the pointwise multiplication because the intersection of two convex curves can be a union of infinitely many singletons.

\section{The Structure of Closed Convex Sets}

The set of finite unions of closed convex sets of a finite-dimensional vector space $V$ over an ordered field is a distributive lattice under the intersection and union of sets. Similarly, the set of finite unions of relatively open convex sets is also a distributive lattice. The elements which are finite unions of compact convex sets in the first lattice are sometimes called polyconvex sets [16]. For this reason, we give the following definition.

Definition 3.1. Let $V$ be a finite-dimensional vector space over $\mathbb{R}$. A subset $E$ of $V$ is called a polyconvex set if it is a finite union of convex sets of $V . E$ is called a closed (relatively open) polyconvex set if the convex sets in the union are closed (relatively open).

Closed (relatively open) polyconvex sets are important geometric objects. In order to get a category for such objects, we need the notion of polyconvex maps.

Definition 3.2. Let $A$ and $B$ be closed (relatively open) polyconvex sets of finite-dimensional vector spaces $V$ and $W$, respectively. A map $f$ from $A$ to $B$ is called a closed (relatively open) polyconvex map if its graph

$$
G(f)=\{(x, f(x)) \mid x \in A\}
$$

is a closed (relatively open) polyconvex set of $V \times W$.

It can be seen that relatively open polyconvex maps need not be continuous. For example, for the relatively open polyconvex sets $A=[0,2] \cup\{3\}$ and $B=[-1,0] \cup[1,2]$, define a map $f(x)=x-1$ for $0 \leq x \leq 1, f(x)=x$ for $1<x \leq 2$, and $f(3)=1$. It can be checked that the graph of $f$ is a finite union of 
relatively open convex sets, so $f$ is a relatively open polyconvex map, but it is not continuous.

Proposition 3.3. The closed (relatively open) polyconvex sets and closed (relatively open) polyconvex maps form a category which is called the category of closed (relatively open) polyconvex sets.

Proof. We just need to show that the composition of polyconvex maps is polyconvex. Let $f$ be a closed (relatively open) polyconvex map from $A$ to $B$ and let $g$ be a closed (relatively open) polyconvex map from $B$ to $C$. Their graphs can be written as

$$
G(f)=\bigcup_{i=1}^{m} E_{i}, \quad G(g)=\bigcup_{j=1}^{n} F_{j}
$$

where $E_{i}$ and $F_{j}$ are closed (relatively open) convex sets. Let the projection images of $E_{i}$ and $F_{j}$ into their first and second components be denoted by $A_{i}, B_{i}, B_{j}^{\prime}$, and $C_{j}$, respectively, then $A_{i}, B_{i}, B_{j}^{\prime}$, and $C_{j}$ are closed (relatively open) convex sets. The convexity of $E_{i}$ and $F_{j}$ implies that $f$ is an affine map from $A_{i}$ to $B_{i}$, and $g$ is an affine map from $B_{j}^{\prime}$ to $C_{j}$. Then $A_{i j}=\left(f \mid A_{i}\right)^{-1}\left(B_{i} \cap B_{j}^{\prime}\right)=A_{i} \cap f^{-1}\left(B_{i} \cap B_{j}^{\prime}\right)$ is convex. Thus the composition map of is affine linear on each $A_{i j}, i=1, \ldots, m$, $j=1, \ldots, n$, and its graph

$$
G(g f)=\bigcup_{i=1}^{m} \bigcup_{j=1}^{n} G_{i j}
$$

is a closed (relatively open) polyconvex set because each $G_{i j}=\left\{(x, f(x)) \mid x \in A_{i j}\right\}$ is a closed (relatively open) convex set.

Proposition 3.4. Let $A$ and $B$ be closed (relatively open) polyconvex sets. If there is a closed (relatively open) polyconvex isomorphism from $A$ to $B$, then $\chi(A)=\chi(B)$.

Proof. Let the graph of the isomorphism $f$ from $A$ to $B$ be written as

$$
G(f)=F_{1} \cup \cdots \cup F_{n},
$$

where each $F_{i}$ is a closed (relatively open) convex set. Let $A_{i}$ and $B_{i}$ be the images of projections of $G(f)$ onto its first and second components. Then $A=A_{1} \cup \cdots \cup A_{n}$ and $B=B_{1} \cup \cdots \cup B_{n} ; A_{i}$ and $B_{i}$ are closed (relatively open) convex sets. Thus for any $i_{1}<\cdots<i_{k}, A_{i_{1}} \cap \cdots \cap A_{i_{k}}$ is nonempty if and only if 
$B_{i_{1}} \cap \cdots \cap B_{i_{k}}$ is nonempty. Therefore

$$
\begin{aligned}
\chi(A) & =\sum_{k=1}^{n}(-1)^{k-1} \sum_{i_{1}<\cdots<i_{k}} \chi\left(A_{i_{1}} \cap \cdots \cap A_{i_{k}}\right) \\
& =\sum_{k=1}^{n}(-1)^{k-1} \sum_{i_{1}<\cdots<i_{k}} \chi\left(B_{i_{1}} \cap \cdots \cap B_{i_{k}}\right)=\chi(B)
\end{aligned}
$$

as desired.

By definition of the category of closed (relatively open) polyconvex sets, it will be noticed that this category has more objects than the maps. This is probably why the algebraic theory of convex sets is not as developed as other geometric objects whose categories have more maps. We prove the structure theorem which has been used in Section 2 for deriving the Euler characteristic of closed and relatively open convex sets.

Proposition 3.5 (Structure Theorem). Let $F$ be a closed convex set in a finitedimensional vector space $V$. Then $F$ has one of the following three forms:

1. $F$ is a compact convex set.

2. $F=K \oplus V^{k}$, where $K$ is a compact convex set and $V^{k}$ is a nonzero $k$ dimensional vector subspace of $V$.

3. $F=M \oplus W \oplus L$, where $W$ is a vector subspace of $V, M$ is a topological manifold (with or without boundary) which is contained in the boundary $\partial \pi(F)$ of $\pi(F)$, where $\pi$ is the canonical projection from $V$ to $V / W$, and $L$ is a closed half-line.

Proof. Let us take an arbitrary point $p$ of $F$ and consider the set

$$
C(p)=\{v \in V \mid p+t v \in F, \forall t \geq 0\}
$$

Then $C(p)$ is a closed convex cone in $V$ with the apex at origin $o$. If $C(p)$ only contains the origin $o$, then $F$ is bounded, so $F$ is a compact convex set, which is the first case. Let $W$ be the largest vector subspace of $V$ which is contained in $C(p)$. If $W$ is not the trivial space $\{o\}$, consider the canonical projection $\pi$ of $F$ onto a subspace $W^{\prime}$, which is a direct complement of $W$ in $V$. Let $K$ be the image of $F$ under the projection $\pi$ in $W^{\prime}$. Then $F=K \oplus W$, and $K$ is a closed convex subset of $W^{\prime}$. If $K$ is compact, then $F$ is the second case. Otherwise, $K$ is unbounded, and, in this case, $K$ does not contain any straight line. Note that there exists a nonzero vector $v$ such that $q+t v \in K$ for all $q \in K$ and for all $t \geq 0$. Let $L=\{t v \mid t \in \mathbb{R}\}$ and let $L_{q}=q+L$ for $q \in K$. Let $M=\bigcup_{q \in \mathbb{R}}\left(\partial L_{q}\right)$, i.e., $M$ is the subset of $\partial K$ whose points are exposed in the opposite direction of $v$. It can be shown that $M$ is a topological manifold with or without boundary. In fact, let $H_{v}$ be any subspace which is a direct complement of $\mathbb{R} v$ in the vector space $\langle K\rangle$ which is spanned by $K$, where $v$ is any 
nonzero vector such that $q+t v \in K$ for all $q \in K$ and $t \geq 0$, and let $\pi_{v}$ be the canonical projection from $\langle K\rangle$ to $H_{v}$. Then $\pi_{v}$ is a homeomorphism from $M$ to $\pi_{v}(K)$, and $\pi_{v}(K)$ is a convex set with or without boundary in $H_{v}$. By our construction we have $F=M \oplus W \oplus L$, which is the desired case three.

Theorem 3.5 can be found in [11]. We do not know of any other references to the structure theorem.

\section{Another Euler Characteristic}

As is mentioned in Section 1, the second component of Schanuel's Euler characteristic for a closed (relatively open) polyconvex set $E$ of $\mathbb{R}^{n}$ can be defined to be the following limit:

$$
\bar{\chi}(E)=\lim _{t \rightarrow+\infty} \chi\left(E \cap I_{t}^{n}\right)
$$

where $I_{t}$ is the closed interval $[-t, t]$ with $t>0, I_{t}^{n}$ is the Cartesian product of $n$ copies of $I_{t}$, and can be replaced by the closed ball

$$
B_{t}=\left\{x \in \mathbb{R}^{n} \mid\|x\| \leq t\right\}
$$

of radius $t$. Since the first component Euler characteristic $\chi$ satisfies the inclusion-exclusion principle, so does $\bar{\chi}$. Then $\bar{\chi}$ can be uniquely extended to a linear functional on $V(\mathscr{F})$ and $V(\mathcal{O})$ by Groemer's extension theorem [5]. We can also define an integral so that $\bar{\chi}$ is well defined on $V(\mathscr{F} \cup \mathcal{O})$ without extension, i.e., for a function $f(x)$ on $\mathbb{R}$ and real numbers $r, s$ such that $r+s=1$, define

$$
\bar{\chi}(f)=\int f(x) d x=\sum_{x \in \mathbb{R}}\left(f(x)-r f\left(x^{-}\right)-s f\left(x^{+}\right)\right),
$$

where $\quad \mathbb{R}=\mathbb{R} \cup\{ \pm \infty\}, \quad f(-\infty)=\lim _{x \rightarrow-\infty} f(x), \quad f(+\infty)=\lim _{x \rightarrow+\infty} f(x)$, $f\left(-\infty^{-}\right)=0, f\left(-\infty^{+}\right)=f(-\infty), f\left(+\infty^{-}\right)=f(+\infty)$, and $\left.f(+\infty)^{+}\right)=0$.

For a function $f(x)$ on $\mathbb{R}^{n}$ and an ordered basis $v=\left\{v_{1}, \ldots, v_{n}\right\}, \bar{\chi}(f, v)$ is defined to be the iterated integral

$$
\bar{\chi}(f, v)=\int f d v_{1} \cdots d v_{n}
$$

$\bar{\chi}(f, v)$ is called the $\bar{\chi}$-integral of $f(x)$ with respect to the basis $v . f$ is called $\bar{\chi}$-integrable if each of the iterated intergrals of $f$ with respect to any basis of $\mathbb{R}^{n}$ is continuous about each coordinate except at finitely many points, and has left and right limits at those discontinuous points. We immediately have the following theorems. 
Theorem 4.1. For any fixed real numbers $r$ and $s$ such that $r+s=1$, each member of the vector space $\mathscr{E}_{r, s}\left(\mathbb{R}^{n}\right)$ is also $\bar{\chi}$-integrable and vice versa.

Theorem 4.2. For each set $E$ of $\mathbb{R}^{n}$, if its indicator function $1_{E}$ is $\bar{\chi}$-integrable, then $\bar{\chi}\left(1_{E}, v\right)$ is an integer for any basis, and is independent of $r$ and $s$ such that $r+s=1$.

A similar structure theorem can occur for relatively open convex sets of $\mathbb{R}^{n}$. In fact, a relatively open convex set $U$ has one of the following three types:

(1) $U$ is bounded,

(2) $U=D \oplus V^{k}$, where $D$ is a bounded relatively open convex set and $V^{k}$ is a nonzero $k$-dimensional subspace of $\mathbb{R}^{n}$, and

(3) $U=M \oplus W \oplus L$, where $M$ is a topological manifold (without boundary) which is contained in $\partial U, W$ is a subspace of $\mathbb{R}^{n}$, and $L$ is an open half-line.

This classification induces the following theorem.

Theorem 4.3. The indicator functions of closed convex sets and relatively open convex sets of $\mathbb{R}^{n}$ are $\bar{\chi}$-integrable. Moreover, for a nonempty closed convex set $F$ and a nonempty relatively open convex set $U$ :

1. $\bar{\chi}(F)=1$.

2. $\bar{\chi}(U)=(-1)^{\operatorname{dim}(U)}$ whenever $U$ is bounded.

3. $\bar{\chi}(U)=(-1)^{\operatorname{dim}(D)}$ whenever $U=D \oplus V^{k}$.

4. $\bar{\chi}(U)=0$ whenever $U=M \oplus W \oplus L$.

The proofs of the above three theorems are omitted because they are essentially the same as the proofs of Theorems $1.3,2.1$, and 2.3. The interested reader can provide similar proofs without difficulty. It should be emphasized that $\bar{\chi}$ is not a topological invariant.

According to the definitions of the Euler integral and $\bar{\chi}$-integral, the two integrals are the same for functions with bounded support. Next we give an example by showing that the compact smooth surface $S_{g}$ of genus $g$ with a standard shape in $\mathbb{R}^{3}$ is an Euler set. The standard means excluding some pathological cases.

We choose a special coordinate so that the handles of the surface face north (z-axis) and are parallel to the $x$-axis. When the indicator function $1_{s_{g}}$ is integrated with respect to $z$ in the direction $\vec{k}$, then $\int 1_{s_{q}} d z$ becomes a function $h$ on $\mathbb{R}^{2}$ whose support is a bounded closed region in $\mathbb{R}^{2}$ with $g$ holes. The function $h$ has a value of 2 in the interior of the closed region and a value of 1 on its boundary. When $h$ is integrated with respect to $y$ in the direction $\vec{j}$, the result is a function $f(x)$ on $\mathbb{R}$ given by

$$
f(x)=\left\{\begin{aligned}
1, & x=a \text { or } b, \\
-1, & x=a_{i} \text { or } b_{i}, \quad 1 \leq i \leq g \\
0, & \text { otherwise }
\end{aligned}\right.
$$


where $a<a_{1}<b_{1}<a_{2}<b_{2}<\cdots<a_{g}<b_{g}<b, a_{i}$ and $b_{i}$ correspond to the image of the boundary of the $i$ th genus on $\mathbb{R}$. So

$$
\chi\left(S_{g}\right)=1+\sum_{i=1}^{g}(-1-1)+1=2-2 g
$$

which is the ordinary Euler characteristic of $S_{g}$. The Euler integral of $1_{s_{g}}$ can be taken with respect to other coordinates, and after a slightly more complicate calculation the same value $2-2 g$ is obtained. There are many other Euler sets in $\mathbb{R}^{n}$, but a general characteristic description is not yet clear. The following Proposition 4.4 is due to a conversation with Dan Klain.

Proposition 4.4. For a function $f$ on $\mathbb{R}^{n}$, if $\chi(f, v)=\chi\left(f, v_{\sigma}\right)$ for all bases $v=$ $\left\{v_{1}, \ldots, v_{n}\right\}$ of $\mathbb{R}^{n}$ and all permutations $\sigma$ of $\{1,2, \ldots, n\}$, where $v_{\sigma}=\left\{v_{\sigma(1)}, \ldots, v_{\sigma(n)}\right\}$, then $\chi(f, v)$ is independent of $v$.

Proof. Fix a basis $u$, any basis $v$ can be obtained by a nonsingular $n$ by $n$ matrix $A$ by $v=u A$. Note that $A$ is a product of some (column) elementary matrices. Then $\chi(f, v)=\chi(f, v A)$ for elementary matrices $A$ and all bases $v$ is equaivalent to $\chi(f, v)$ being independent of $v$. It is trivial to check that $\chi(f, v)=\chi(f, v A)$ by definition whenever $v A$ is $\left\{v_{1}, \ldots, a v_{i}, \ldots, v_{n}\right\}$ or $\left\{v_{1}, \ldots, v_{i}, \ldots, v_{j}+a v_{i}, \ldots, v_{n}\right\}$ for a nonzero number $a$. Thus the proposition follows immediately.

In order to get a useful sufficient condition we need the notion of coordinately cellular sets which are inductively defined in the following.

\section{Definition 4.5.}

(1) Every open interval or a singleton in a one-dimensional subspace $L$ is a cell with respect to any basis of $L$.

(2) Let $v=\left\{v_{1}, \ldots, v_{n}\right\}$ be a basis of $\mathbb{R}^{n}$. Let $H_{i}$ be the subspace spanned by $\left\{v_{1}, \ldots, v_{i-1}, v_{i+1}, \ldots, v_{n}\right\}$ and let $p_{i}$ be the canonical projection from $\mathbb{R}^{n}$ to $H_{i}(1 \leq i \leq n)$. A subset $C$ of $\mathbb{R}^{n}$ is a cell with respect to $v$ if $p_{i}(C)$ is a cell with respect to the basis $\left\{v_{1}, \ldots, v_{i-1}, v_{i+1}, \ldots, v_{n}\right\}$ of $H_{i}$ and $p_{i}^{-1}(x) \cap C$ is simultaneously an open interval or a singleton for each $x \in p_{i}(C)$.

(3) A subset $E$ of $\mathbb{R}^{n}$ is called coordinately cellular if, for each basis $v$ of $\mathbb{R}^{n}, E$ can be decomposed into a disjoint union of cells with respect to $v$.

Theorem 4.6. Every indicator function of a coordinately cellular set of $\mathbb{R}^{n}$ is Euler integrable, and its Euler integral is independent of the choice of bases.

Proof. It is obvious that every coordinately cellular set $E$ is Euler integrable. For each basis $v=\left\{v_{1}, \ldots, v_{n}\right\}$ of $\mathbb{R}^{n}$, by definition, a cell structure $\left\{C_{i}\right\}$ of $E$ with respect to $v$ is also a cell structure with respect to the basis

$$
v_{\sigma}=\left\{v_{\sigma(1)}, \ldots, v_{\sigma(n)}\right\}
$$


for any permutation $\sigma$ of $\{1,2, \ldots, n\}$. Thus

$$
\chi(E, v)=\sum_{i}(-1)^{\operatorname{dim}\left(C_{i}\right)}=\chi\left(E, v_{\sigma}\right)
$$

The conclusion follows from Proposition 4.4 .

\section{Remarks}

The usual Euler integral can be referred to as the $\chi$-integral. It is natural to ask whether the Euler integrals can be carried out on some more general spaces such as compact smooth manifolds by transversal foliations, Lie groups by independent left or right invariant vector fields, and projective spaces by projective lines. It will be very interesting and important to characterize the functions of $\mathscr{E}_{r, s}\left(\mathbb{R}^{n}\right)$ by some equivalent conditions, or even by some sufficient conditions. A possible condition for the characterization of Euler sets is asked in the following question.

Question 5.1. Is it true that a subset of $\mathbb{R}^{n}$ is an Euler set if and only if it is a coordinately cellular set?

It can be proved that closed convex sets, relatively open convex sets, semialgebraic sets, and finitely subanalytic sets are all coordinately cellular. By the calculation of Euler integrals of some simple functions, it will be noticed that the basis chosen is not essential. This suggests the following weak version of Question 5.1.

Question 5.2. If $E$ is an Euler set of $\mathbb{R}^{n}$, is it true that the Euler integral of its indicator function $1_{E}$ with respect to any basis has same value?

Since curvature measures for finite unions of compact convex sets are constructed by the Euler characteristic, similar curvature measures for members of $V(\mathscr{F} \cup \mathcal{O})$ with compact support can be constructed by our Euler characteristic. Moreover, the Minkowski addition and related algebraic theory about $V(\mathscr{F})$ and $V(\mathcal{O})$ can also be obtained by the Euler characteristic.

\section{Acknowledgments}

I wish to thank Dan Klain, Gian-Carlo Rota, Steve Schanuel, and the referees for their helpful discussions and suggestions. 


\section{References}

1. M. Cerasoli and G. Letta, Sulla caratteristica d'Euler-Poincaré, Rend. Acad. Naz. Sci. XII Mem. Mat. 106 (1988), fasc. 18, 259-267.

2. B. F. Chen, The Gram-Sommerville and Gauss-Bonnet theorems and combinatorial geometric measures for noncompact polyhedra, Adv. in Math. 91 (1992), 269-291.

3. H. Groemer, Eulersche Charakteristik, Projektionen und QuermaB-integrale, Math. Ann. 198 (1972), 23-56.

4. H. Groemer, On the Euler characteristic and related functionals on convex surfaces, Geom. Dedicata 4 (1975), 91-104.

5. H. Groemer, Minkowski addition and mixed volumes, Geom. Dedicata 6 (1977), 141-163.

6. H. Groemer, On the extension of additive functionals on classes of convex sets, Pacific J. Math. 75 (1978), 397-410.

7. H. Groemer, On rings of sets associated with characteristic functions, Arch. Math. (Basel), 49 (1987), 91-93.

8. H. Hadwiger, Eulers Charakteristik und kombinatorische Geometrie, J. Reine Angew. Math. 194 (1955), 101-110.

9. H. Hadwiger and H. P. Mani, On the Euler characteristic of spherical polyhedra and the Euler relation, Mathematika 19 (1972), 139-143.

10. V. Klee, The Euler characteristic in combinatorial geometry, Amer. Math. Monthly 70 (1963), 119-127.

11. W. Ledermann and S. Vajda, Combinatorics and Geometry, Handbook of Applicable Mathematics, Vol. V, Part A, pp. 105-137.

12. P. McMullen and R. Schneider, Valuations of convex bodies, in Convexity and Its Applications (P. M. Gruber and J. M. Wills, eds.), pp. 170-247, Birkhäuser, Boston, 1983.

13. R. Morelli, A theory of polyhedra, Adv. in Math. 97 (1993), 1-73.

14. G.-C. Rota, On the foundations of combinatorial theory, I. Theory of Möbius functions, Z. Wahrsch. 2 (1964), 340-368.

15. G.-C. Rota, On the combinatorics of the Euler characteristic, in Studies in Pure Mathematics (L. Mirsky, ed.), pp. 221-233, Academic Press, London, 1971.

16. G.-C. Rota, Introduction to geometric probability, Lezioni Lincee held at the Scuola Normale Superiore, Pisa, Dec. 2-22, 1986.

17. S. Schanuel, What Is the Length of a Potato? An Introduction to Geometric measure Theory, Lecture Notes in Mathematics, Vol. 1174, pp. 118-126, Springer-Verlag, Berlin, 1986.

18. S. Schanuel, Negative Sets have Euler Characteristic and Dimension, Lecture Notes in Mathematics, Vol. 1488, Springer-Verlag, Berlin, 1991.

Received January 16, 1992, and in revised form July 24, 1992. 